\title{
Interstitial pneumonitis with autoimmune features (IPAF): a work in progress
}

\author{
Fabrizio Luppi ${ }^{1}$ and Athol U. Wells ${ }^{2}$ \\ Affiliations: ${ }^{1}$ Center for Rare Lung Diseases, University Hospital of Modena, Modena, Italy. ${ }^{2}$ Interstitial Lung \\ Diseases Unit, Royal Brompton Hospital, London UK.
}

Correspondence: Athol U. Wells, Royal Brompton Hospital, Sydney St, London, SW3 6HP, UK.

E-mail: rbhildarbht.nhs.uk

0

@ERSpublications

For the IPAF definition to be accepted, it needs to be shown to add information on the likely future disease course http://ow.ly/10zy1P

In clinical practice, it is common to encounter patients with an "idiopathic" interstitial pneumonia (IIP) associated with features suggestive of, but not diagnostic for, a classical connective tissue disease (CTD). In some cases, serological abnormalities are absent and in other cases, serological findings are suggestive of a specific CTD, but systemic clinical features are not. Alternatively, thoracic radiological or histopathological features are compatible with or highly suggestive of an underlying CTD, but diagnostic criteria built on serological and systemic clinical findings are not met. In suspected idiopathic pulmonary fibrosis (IPF), in particular, the designation of an underlying autoimmune process has profound management implications. The medical literature is punctuated by statements and series exploring aspects of this demi-semi-syndrome, variously termed "undifferentiated CTD associated interstitial lung disease" (UCTD-ILD), "lung-dominant CTD" and "autoimmune-featured ILD" [1-4]. However, the absence of standardised criteria has hindered progress and a new beginning was necessary. In a recent European Respiratory Society/American Thoracic Society research statement [5], the term "interstitial pneumonia with autoimmune features" (IPAF) was proposed to describe the presence of a combination of features from any two of three domains: a clinical domain, consisting of specific extrathoracic features; a serological domain, consisting of specific autoantibodies; and a morphological domain, consisting of specific chest imaging, histopathological or pulmonary physiological features.

OlDhAm et al. [6] retrospectively applied IPAF criteria to a cohort of 422 patients diagnosed with IIP, interstitial pneumonia associated with UCTD or unclassifiable ILD. The finding that approximately one-third of the initial cohort $(\mathrm{n}=144)$ met IPAF criteria confirmed earlier reports $[4,7,8]$ that features of CTD are common in patients with IIP. Morphological and serological domain criteria were both satisfied in over three-quarters of IPAF patients (including a significant subgroup satisfying criteria for all three domains). Mortality in patients meeting IPAF criteria was marginally lower than in patients with IPF, and higher than in patients with CTD-ILD.

This outcome difference was primarily linked to underlying radiographic and/or histological patterns: IPAF patients with non-usual interstitial pneumonia (UIP) patterns $(n=46)$ had a higher survival, which did not differ significantly from that in CTD-ILD, whereas the outcome in IPAF patients with a UIP pattern $(n=98)$ was similar to that in IPF. Within IPAF patients, satisfaction of the clinical domain was associated with increased survival, whereas satisfaction of the serological or morphological domains were both associated with a marginal increase in mortality. Interestingly, the presence of multi-compartment disease was a malignant prognostic determinant.

Based on these and other findings, a subgroup of patients was designated in which clinical domain criteria were met and a non-UIP pattern at high-resolution computed tomography (HRCT) or surgical lung

Received: April 052016 | Accepted: April 062016

Conflict of interest: Disclosures can be found alongside this article at erj.ersjournals.com

Copyright OERS 2016 
biopsy (SLB) was present (i.e. serological data and the presence or absence of multi-compartment disease were not taken into account). Survival mirrored that in CTD-ILD, but this subgroup consisted of 40 of the 46 IPAF patients with non-UIP patterns, who were also characterised by a CTD-ILD-like outcome. Due to major subgroup overlap of this sort and the lack of powering for other outcome analyses, the nuanced prognostic distinctions reported by OlDHAM et al. [6] will need to be tested in future cohorts. What can be concluded with confidence is that, within patients meeting IPAF criteria, the distinction between a UIP and a non-UIP pattern, whether on HRCT or biopsy, has major prognostic significance. Once this has been taken into account, it is not altogether clear from these data that an IPAF designation in an IIP patient provides added prognostic value.

The criteria used to define IPAF are, in the final analysis, based on expert opinion and need to be revisited as further data are accumulated. This fact should not be viewed as a weakness of the IPAF initiative: a new classification or diagnostic entity cannot, by definition, be justified solely by the existing evidence-base. The IPAF criteria, including the need to satisfy at least two of the morphological, serological and systemic clinical domains, were based on the fact that this framework "makes sense" to expert rheumatologists, chest physicians, radiologists and histopathologists. The process of reasoned debate without definitive evidential proof has, in the past, underpinned major changes in the definition of disease, including the formulation of the classical CTDs. However, concepts that "make sense" do not always stand the test of time and it is conceivable that individual IPAF criteria may eventually need to be radically refined.

A single example may suffice: the category of multi-compartment disease within the morphological domain. In predominantly restrictive ILD, it is often obvious that there is coexisting airflow obstruction or disproportionate pulmonary vasculopathy. However, in many other patients, there is considerable uncertainty as to whether an element of airflow obstruction or marginal evidence of vasculopathy is sufficient to justify a multi-compartment designation. Should the presence of coexisting airway disease be based primarily on pulmonary function tests, morphological findings (on HRCT or at biopsy), or a composite of HRCT, biopsy and lung function variables (as used by Oldham et al. [6])? Is the forced expiratory volume in $1 \mathrm{~s} /$ forced vital capacity ratio of 0.70 , applied by OLDHAM et al. [6], "fit for use" in the context of a predominantly restrictive process or would a measure such as the residual volume/total lung capacity ratio, denoting obstruction in the presence of restriction, be preferable? Must pulmonary hypertension be present in order to establish that there is a vasculopathic component and if so, how should it be diagnosed? Or is it sufficient that measures of gas transfer should be selectively or disproportionately impaired when compared with lung volumes, as used in the study of Oldham et al. [6]? Whatever measure of airflow obstruction is chosen, how should this be adjusted according to the presence or absence of emphysema on HRCT?

There is an obvious means of exploring these uncertainties in future studies. Intrinsic to the assumptions underlying the creation of the IPAF entity is the belief that there is clustering between the clinical, serological and morphological features. But is this truly the case and if so, can the association between multi-compartment disease and other IPAF features be used to refine the definition of the airway and vasculopathic components? Certainly, an association between multi-compartment disease and IPAF itself is not informative as the argument is circular: many patients satisfy IPAF criteria because multi-compartment disease is believed to be present. OLDHAM et al. [6] state that clustering between IPAF features did exist in their cohort but the devil is in the detail. In principle, it should be possible to examine thresholds used to identify airway or vascular disease against key IPAF features in other domains, such as Raynaud's phenomenon. In a broader cohort such as that studied by OLDHAM et al. [6], it can be argued that the most robust definition of multi-compartment disease should be the definition most strongly associated with non-morphological IPAF features. This approach, analogous to the evaluation of "construct validity" across domains in the development of trial end-points, may be highly informative.

Ongoing uncertainties exist in the clinical domain of IPAF, not least with regard to the role of specialist rheumatological assessment. In at least one European country, a formal protocol requires the presence of a rheumatologist whenever a multidisciplinary ILD diagnosis is made. However, the skills of a rheumatologist lie in the evaluation of the patient (and not in being informed by a respiratory physician in a multidisciplinary meeting that systemic rheumatological features are present!). For example, while an inflammatory arthropathy is sometimes clinically overt, in many other cases the distinction between osteoarthritis and a true inflammatory phenotype lies beyond the competence of a respiratory physician. By contrast, assessment of the intrathoracic morphological domain of IPAF, requiring the integration of radiological findings, histological features, pulmonary function data and the clinical features of lung disease, lies beyond the competence of the rheumatologist. The designation of IPAF requires independent conclusions to be reached on each IPAF domain and there is no overlap in this process between the skills of a rheumatologist and the skills of other sub-specialists. An alternative model in which patients with suspected IPAF are evaluated jointly by a rheumatologist and a respiratory physician in a CTD-ILD clinic has obvious advantages. 
Another potentially important clinical nuance is the anecdotal observation that there is sometimes temporal clustering between the development of lung disease and the onset of a number of systemic features suggestive of autoimmunity. The simultaneous or near-simultaneous development of IPAF features in two domains must surely increase the likelihood of biological linkage between these features. This may also apply to clinical features (such as sicca symptoms and gastro-oesophageal reflux) that are compatible with, but less specific for, autoimmune disease and are excluded from the IPAF clinical domain. If, for example, the development of nonspecific interstitial pneumonia occurs concurrently with the onset of severe sicca symptoms and striking gastro-oesophageal reflux, the thinking clinician is likely to integrate these features, increasing the perceived likelihood of an autoimmune pathogenesis. This logic is not endorsed by the IPAF statement for good reason: as discussed earlier, the IPAF entity is necessarily "speculative" at this stage and attempting to integrate anecdotal clinical nuances would be over-speculative. However, the importance of temporal clustering of thoracic and systemic clinical features needs to be explored.

These uncertainties aside, the pivotal question, explored by OLdHAm et al. [6] is whether IPAF might have prognostic significance. The creation of a diagnostic entity can be justified either by attendant pathogenetic insights (potentially leading to treatment advances) or clinical utility. Accurate diagnosis is of value primarily because it informs the clinician of the likely natural history and treated course. Evidence that IPAF designation is, on average, associated with a significant change in expected outcome would provide ample validation of the formal integration of IPAF into ILD diagnostic classifications. The analyses of OLDHAM et al. [6] suggest that, as currently defined, a designation of IPAF may not provide important prognostic value over and above HRCT and biopsy evaluation. However, there are two important caveats. First, a surprisingly high proportion of patients in this cohort had undergone a SLB and it is difficult, therefore, to relate these findings with confidence to the large subgroup of non-biopsied patients managed by most ILD physicians. The clinical and serological IPAF domains may have greater prognostic significance in patients with "possible UIP" on HRCT, in whom, for one reason or another, a SLB is impracticable. Secondly, evaluation of the IPAF clinical domain by a specialist rheumatologist and refinement of the definition of multi-compartment disease may be vitally important in identifying clinically significant outcome differences, especially in patients with known or suspected UIP. Thus, the findings of Oldham et al. [6] are valuable mostly because they highlight existing uncertainties that need to be addressed in the years to come.

\section{References}

1 Fischer A, West SG, Swigris JJ, et al. Connective tissue disease-associated interstitial lung disease: a call for clarification. Chest 2010; 138: 251-256.

2 Kinder BW, Collard HR, Koth L, et al. Idiopathic nonspecific interstitial pneumonia: lung manifestation of undifferentiated connective tissue disease? Am J Respir Crit Care Med 2007; 176: 691-697.

3 Vij R, Noth I, Strek ME. Autoimmune-featured interstitial lung disease: a distinct entity. Chest 2011; 140: 1292-1299.

4 Corte TJ, Copley SJ, Desai SR, et al. Significance of connective tissue disease features in idiopathic interstitial pneumonia. Eur Respir J 2012; 39: 661-668.

5 Fischer A, Antoniou KM, Brown KK, et al. An official European Respiratory Society/American Thoracic Society research statement: interstitial pneumonia with autoimmune features. Eur Respir J 2015; 46: 976-987.

6 Oldham JM, Adegunsoye A, Valenzi E, et al. Characterisation of patients with interstitial pneumonia with autoimmune features. Eur Respir J 2016; 47: 1767-1775.

7 Assayag D, Kim EJ, Elicker BM, et al. Survival in interstitial pneumonia with features of autoimmune disease: a comparison of proposed criteria. Respir Med 2015; 109: 1326-1331.

8 Ferri C, Manfredi A, Sebastiani M, et al. Interstitial pneumonia with autoimmune features and undifferentiated connective tissue disease: our interdisciplinary rheumatology-pneumology experience, and review of the literature. Autoimmun Rev 2016; 15: 61-70. 